\title{
Structural Elucidation on Succinoglycan and Related Polysaccharides from Agrobacterium and Rhizobium by Fragmentation with Two Special $\beta$-D-Glycanases and Methylation Analysis
}

\author{
Makoto Hisamatsu, Jun-ichi Abe, Akinori Amemura \\ and Tokuya Harada \\ Institute of Scientific and Industrial Research, \\ Osaka University, Suita, Osaka 565, Japan \\ Received October 5, 1979
}

\begin{abstract}
The structure of the extracellular acidic polysaccharide succinoglycan from Alcaligenes faecalis var. myxogenes $10 \mathrm{C} 3$ was elucidated by successive fragmentation of the polysaccharide with extracellular $\beta$-D-glycanase (succinoglycan depolymerase) and intracellular endo- $\beta$-( $1 \rightarrow 6)$-Dglucanase of Flavobacterium sp. M64 into two tetrasaccharides via its octasaccharide repeating unit, and then methylation analysis and enzymic hydrolysis of the products. The extracellular acidic polysaccharides from Rhizobium meliloti, Agrobacterium radiobacter, Agrobacterium rhizogenes and Agrobacterium tumefaciens were also analyzed by this method and found io be identical with the polysaccharide from Alcaligenes faecalis var. myxogenes, irrespective of their modes of acylation. The structure was identical with that of extracellular polysaccharide from Rhizobium meliloti presented by Jansson et al. (J. Am. Chem. Soc., 99, 3812 (1977)), except for the occurrence of the succinic acid residue.
\end{abstract}

Succinoglycan, an extracellular acidic polysaccharide produced by Alcaligenes faecalis var. myxogenes $10 \mathrm{C} 3$, contains D-glucose, Dgalactose and succinic acid. ${ }^{2)}$ Recently, pyruvic acid was also found in this polysaccharide. ${ }^{3)}$ The polysaccharide moiety consists of $(1 \rightarrow 3)$-, $(1 \rightarrow 4)$-, $(1 \rightarrow 6)$ - and $(1 \rightarrow 4,1 \rightarrow 6)$-linked $\beta$-Dglucose residues and $(1 \rightarrow 3)$-linked $\beta$-Dgalactose residues. ${ }^{4.5)}$ The succinic acid residues are attached to the polysaccharide chain by ester linkages. ${ }^{2)}$ Many strains of Agrobacterium were also shown to form a watersoluble acidic polysaccharide like succinoglycan. ${ }^{3)}$ Methylation analyses of the polysaccharides from Agrobacterium tumefaciens and Rhizobium meliloti gave similar results to those for succinoglycan. ${ }^{6}$ Jansson et al. ${ }^{11}$ established the structure of the extracellular polysaccharide from Rhizobium meliloti by a method involving methylation and specific sequential degradation." Comparative studies on the polysaccharides from Rh. meliloti, Al. faecalis var. myxogenes and Agrobacterium radiobacter by methylation, ${ }^{13} \mathrm{C}$-NMR and enzyme degra- dation provided strong evidence that these polysaccharides have identical structures, irrespective of their modes of acylation. ${ }^{7 /}$

We have been studying two $\beta$-D-glycanases formed by Flavobacterium sp. M64, a strain isolated from soil as an organism capable of growing with succinoglycan as the sole source of carbon. ${ }^{8)}$ One is an extracellular $\beta$-Dglycanase, succinoglycan depolymerase, which hydrolyzes succinoglycan into octasaccharide repeating unit, ${ }^{9)}$ and the other is an intracellular endo- $\beta-(1 \rightarrow 6)$-D-glucanase which hydrolyzes the repeating unit into two tetrasaccharides. ${ }^{10)}$ We now report a method involving fragmentation with these glycanases and methylation analysis for determining the structure of the complicated $\beta$-D-glycan. The fine structure of succinoglycan was elucidated by this method.

\section{MATERIALS AND METHODS}

Organisms. Alcaligenes faecalis var. myxogenes $10 \mathrm{C} 3$, which was isolated from soil as an organism capable of 
growing in the medium containing $10 \%$ ethyleneglycol as the sole carbon source, ${ }^{11}$ Agrobacterium radiobacter IFO 12665, Agrobacterium rhizogenes IFO 13259 and Agrobacterium tumefaciens IFO 3058 were used for production of extracellular acidic polysaccharides.

Preparation of extracellular acidic polysaccharides, and products of the polysaccharides by enzymatic hydrolysis. Extracellular acidic polysaccharides were prepared from the supernatants of cultures grown in synthetic medium containing $4 \%$ glucose as described previously. ${ }^{3.12)}$ The acidic polysaccharide from Rhizobium meliloti U27 was supplied by Prof. B. Lindberg. An octasaccharide (SG-D) which represents repeating unit was prepared by hydrolysis of the polysaccharides with succinoglycan depolymerase, an extracellular $\beta$-D-glycanase of Flavobacterium sp. M64, as described previously. "The two tetrasaccharides (desuccinylated oligosaccharide I and oligosaccharide II) were prepared by hydrolysis of the desuccinylated SG-D with the intracellular $\beta$-D-glycanase of Flavobacterium sp. M64. ${ }^{101}$ The desuccinylated SG-D was used as the substrate for the intracellular $\beta$-D-glycanase, since it was not easy to separate the two tetrasaccharides (oligosaccharide I and II) derived from the enzymatic hydrolysate of SG-D by paper chromatography. ${ }^{109}$ Oligosaccharide $I$ is composed of D-glucose, pyruvic acid and succinic acid $(4: 1: 1$, molar ratio) and oligosaccharide II is composed of D-glucose and D-galactose $\left(3: 1\right.$, molar ratio) ${ }^{10)}$

Methylation analysis. Samples $(0.5 \sim 2.0 \mathrm{mg})$ were methylated by the method of Hakomori ${ }^{13)}$ with sodium methylsulfinyl methanide, and methyl iodide in methyl sulfoxide. The methylated polysaccharide was first treated with $1 \mathrm{ml}$ of $90 \%$ formic acid for $6 \mathrm{hr}$ at $100^{\circ} \mathrm{C}$ in a sealed tube under nitrogen and then evaporated in vacuo. Hydrolysis was continued in $0.5 \mathrm{ml}$ of $2 \mathrm{M}$ trifluoroacetic acid for $6 \mathrm{hr}$ at $100^{\circ} \mathrm{C}$ in a sealed tube under nitrogen. The sample of methylated oligosaccharide was hydrolyzed directly with $2 \mathrm{M}$ trifluoroacetic acid. The hydrolysates were analyzed as alditol acetates in a Shimadzu GC 7A gas chromatograph, fitted with a flame ionization detector and a column $(3 \mathrm{~mm} \times 2 \mathrm{~m}$ ) of $0.3 \%$ OV $275-0.4 \%$ GEXF 1150 on Shimalite $\mathrm{W}(80 \sim 100$ mesh $)$. The column temperature was held for $4 \mathrm{~min}$ at $120^{\circ} \mathrm{C}$ and then raised at $1{ }^{\circ} \mathrm{C} / \mathrm{min}$ to $180^{\circ} \mathrm{C}$. Nitrogen was used as a carrier, at a flow rate of $50 \mathrm{ml} / \mathrm{min}$

The GLC pattern of the methylated sugars from depyruvylated succinoglycan is shown in Fig. 1. Peaks A, B, D, E and $F$ were identified as the alditol acetates of 2,3,4,6-tetra$O$-methyl-D-glucose, 2,4,6-tri- $O$-methyl-D-glucose, 2,3,4tri- $O$-methyl-D-glucose, 2,3,6-tri- $O$-methyl-D-glucose and 2,3-dj- $O$-methyl-D-glucose, respectively, by comparison with partially methylated alditol acetates derived from laminarabiose, gentiobiose, maltose and glycogen. Peak C was collected from the effluent gas in a capillary glass tube and identified as the alditol acetate of 2,4.6-tri- $O$-methyl-Dgalactose $(T=2.28)$ with the $3 \%$ ECNSS-M column. $\left.{ }^{14}\right)$
The column of $0.3 \%$ OV275-0.4\% GEXF1150 was useful for clear separation of these peaks and was excellent for analysis of the methylated sugars from polysaccharides such as succinoglycan.

Determination of reducing terminal sugar of the octasaccharide repeating unit ( $S G-D)$. SG-D $(2 \mathrm{mg})$ obtained by hydrolysis of succinoglycan with succinoglycan depolymerase was dissolved in $2 \mathrm{ml}$ of water and reduced by treatment with sodium borohydride $(4 \mathrm{mg})$. The reduced sample was hydrolyzed in a sealed tube with $1 \mathrm{ml}$ of $2 \mathrm{M}$ hydrochloric acid for $2 \mathrm{hr}$ at $100^{\circ} \mathrm{C}$ under nitrogen. The hydrolysate was acetylated and analyzed in a Shimadzu GC 7A gas chromatograph at a gas flow rate of $60 \mathrm{ml} / \mathrm{min}$ at $190^{\circ} \mathrm{C}$. The acetyl derivatives of sugar and sugar alcohol were separated on a column $(3 \mathrm{~mm} \times 2 \mathrm{~m})$ packed with $1.5 \%$ Silicon OV-17 on Shimalite $W^{15)}$ Sugar alcohols were collected from the effluent gas by condensing them in capillary glass tubes attached to the exit port of the gas chromatograph, and were dissolved in chloroform and rechromatographed on a column packed with $3 \%$ ECNSS$\mathrm{M}$ on Uniport Hp.

Preparation of desuccinylated succinoglycan and the desuccinylated $S G-D$. A solution of $0.1 \%$ succinoglycan or SG-D was stirred in $10 \mathrm{~mm}$ potassium hydroxide for $5 \mathrm{hr}$ at $20^{\circ} \mathrm{C}$ under nitrogen as described by Sloneker and Jeanes. ${ }^{16)}$

Preparation of depyruvylated succinoglycan, depyruvylated $S G-D$ and depyruvylated, desuccinylated oligosaccharide $I$. A solution of $0.1 \%$ succinoglycan, SG-D or desuccinylated oligosaccharide I was heated in $10 \mathrm{~mm}$ hydrochloric acid for $90 \mathrm{~min}$ at $100^{\circ} \mathrm{C}$ under nitrogen. This method is a modification of the procedure of Chaudhari $e t$ al. ${ }^{17)}$

Hydrolysis of oligosaccharide $I$ by $\beta$-D-glucosidase of almond. Depyruvylated, desuccinylated oligosaccharide 1 $(0.2 \mathrm{mg})$ was digested with almond $\beta$-D-glucosidase $(20 \mu \mathrm{g}$, Sigma Chemical Co.) for $2 \mathrm{hr}$ at $40^{\circ} \mathrm{C}$ in $100 \mu 1$ of $50 \mathrm{~mm}$ sodium acetate buffer $(\mathrm{pH} 5.0)$. The mixture was then boiled for $1 \mathrm{~min}$, applied to Toyo-Roshi No. 50 paper and developed with a solvent system of 1-butanol-pyridinewater $(6: 4: 3, v / v)$. Sugars were detected on paper chromatograms with silver nitrate reagent.

Digestion of side chain of succinoglycan by $\beta$-Dglucosidase of almond. Depyruvylated, desuccinylated succinoglycan $(5 \mathrm{mg})$ was digested from the non-reducing terminal sugar of the side chain with almond $\beta$-Dglucosidase $(5 \mathrm{mg})$ for 3 days at $30^{\circ} \mathrm{C}$ in $0.5 \mathrm{ml}$ of $50 \mathrm{~mm}$ sodium acetate buffer ( $\mathrm{pH}$ 5.0). One drop of toluene was added to prevent microbial growth. The mixture was then boiled for $1 \mathrm{~min}$, the resulting precipitate was removed, and the supernatant was dialyzed. 


\section{RESULTS AND DISCUSSION}

Succinoglycan was hydrolyzed completely into the octasaccharide repeating unit (SGD) with succinoglycan depolymerase. ${ }^{93}$ Desuccinylated SG-D was further hydrolyzed completely with the intracellular endo- $\beta$ $(1 \rightarrow 6)$-D-glucanase of Flavobacterium sp. M64 into two tetrasaccharides (desuccinylated oligosaccharide I and oligosaccharide II). ${ }^{10}$ Succinoglycan, depyruvylated succinoglycan, SG-D, depyruvylated SG-D, desuccinylated oligosaccharide I, depyruvylated, desuccinylated oligosaccharide I and oligosaccharide II were methylated and the methylated sugars were analyzed as alditol acetates by GLC on a column of $0.3 \%$ OV $275-0.4 \%$ GEXF 1150 as shown in Table I. The GLC pattern of depyruvylated succinoglycan is shown in Fig. 1.

Methylation data on succinoglycan and depyruvylated succinoglycan show that succinoglycan has glucose residues branching through a $(1 \rightarrow 4)$ - or $(1 \rightarrow 6)$-linkage and non-reducing terminal glucose residues with pyruvic acid linked to $O-4$ and $O-6$; these give 2,3-di-Omethyl-D-glucose. The same pattern of methylated sugar is obtained with succinoglycan and desuccinylated succinoglycan because an ester linkage between succinic acid and sugar residue is hydrolyzed during the methylation procedure. Thus, methylation analysis is unable to afford any information about the binding site of succinic acid.

Methylation analyses of SG-D and depyruvylated SG-D indicate that SG-D has no branching residues. Thus, succinoglycan depolymerase hydrolyzes the linkages adjacent to branching glucose residues to produce linear repeating units. These linkages must be $(1 \rightarrow 4)$, because there was an increase of $1 \mathrm{~mol}$ of 2,3,4tri- $O$-methyl-D-glucose, corresponding to $(1 \rightarrow 6$ )-linked D-glucose, on hydrolysis with succinoglycan depolymerase.

For determination of the reducing terminal sugar of SG-D, SG-D was reduced with sodium borohydride and hydrolyzed with hydrochloric acid, and the hydrolysate was analyzed as acetyl derivatives by GLC on a column of Silicone OV-17. In the resulting chromatogram shown in Fig. 2 peaks $A$ and B correspond to $\alpha$ and $\beta$-D-glucose pentaacetate, respectively, (retention times relative to $\mathrm{D}$-glucitol hexaacetate, 0.65 and 0.72 , respectively), and peak $\mathrm{C}$ corresponds to $\mathrm{D}$-glucitol hexaacetate or $\mathrm{D}$ -

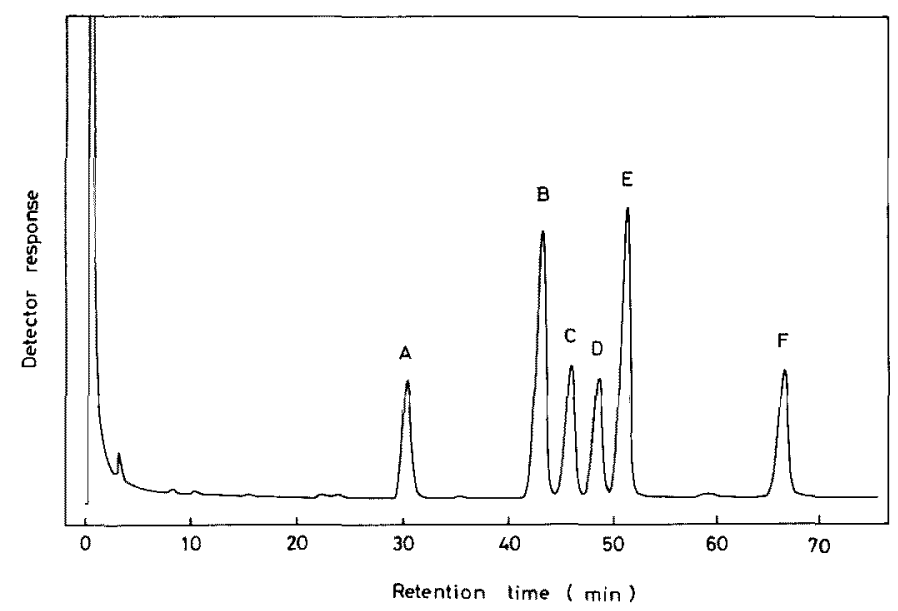

FiG. 1. GLC Pattern of the Alditol Acetates of Methylated Sugars from Depyruvylated Succinoglycan on an OV275-GEXF1150 Column.

(A) 1,5-di- $O$-acetyl-2,3,4,6-tetra- $O$-methyl-D-glucitol; (B) 1,3,5-tri- $O$-acetyl-2,4,6-tri- $O$-methyl-D-glucitol; (C) 1,3,5-tri- $O$-acetyl-2,4,6-tri- $O$-methyl-D-galactitol; (D) $1,5,6$-tri- $O$-acetyl-2,3,4-tri- $O$-methyl-D-glucitol; (E) 1,4,5-tri- $O$-acetyl-2,3,6-tri- $O$-methyl-D-glucitol; (F) 1,4,5,6-tetra- $O$-acetyl-2,3-di- $O$-methyl-D-glucitol. 


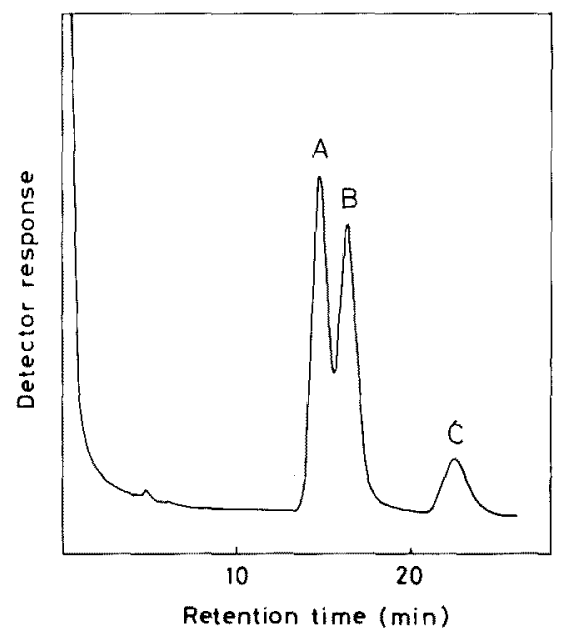

FlG. 2. GLC Pattern of the Acetyl Derivatives of an Acid Hydrolysate of Reduced Octasaccharide (SG-D) on an OV17 Column.

(A) $\alpha$-D-glucose pentaacetate; (B) $\beta$-D-glucose pentaacetate; (C) D-glucitol hexaacetate or D-galactitol hexaacetate.

galactitol hexaacetate, since these two compounds were not separated on this column. For identification of peak $C$, this fraction was collected from the effluent gas and rechroma- tographed on a column of ECNSS-M. As shown in Fig. 3, a single peak corresponding to Dgalactitol hexaacetate was obtained (retention time relative to $\mathrm{D}$-glucitol hexaacetate, 0.87 ). Thus the reducing terminal sugar of SG-D was identified as D-galactose. From Fig. 2, the ratio of glucose to sugar alcohol (galactitol) is 7, and thus the degree of polymerization of the repeating units of succinoglycan is 8 . Previously, we reported $^{9)}$ that the degree of polymerization of SG-D was 12 , but we noticed that the preparation contained a small amount of an impurity with glucose as the reducing terminal.

Desuccinylated oligosaccharide I was composed of two $(1 \rightarrow 3)$-linked D-glucose residues, one $(1 \rightarrow 6)$-linked $D$-glucose residue and one non-reducing terminal $D$-glucose residue with pyruvic acid (Table I). When depyruvylated, desuccinylated oligosaccharide I was digested from the non-reducing terminal with almond $\beta$ D-glucosidase gentiobiose was detected as the sole disaccharide (Fig. 4), indicating that the sequence of reducing part of the oligosaccharide is $\mathrm{D}-\mathrm{Glc} P-(1 \rightarrow 6)-\mathrm{D}-\mathrm{Glc} P$. These results indicate that the structure of desuccinylated oligosaccharide I is

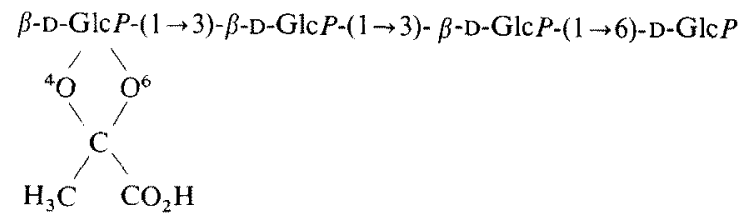

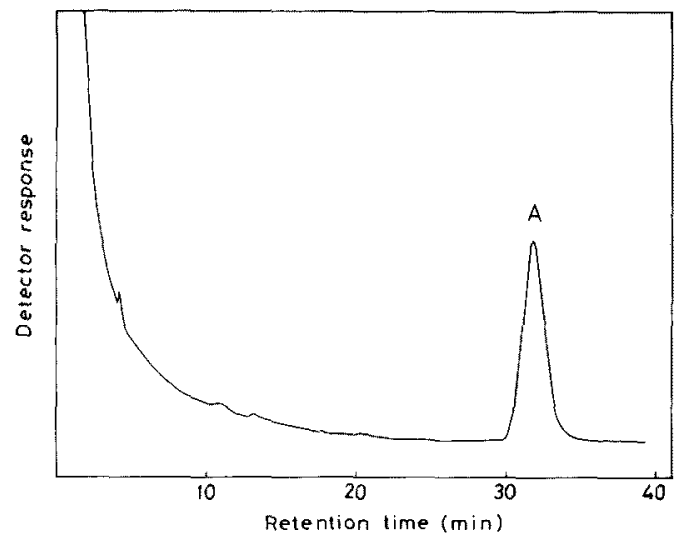

FIG. 3. GLC Pattern of Peak C in Fig. 2 on an ECNSS-M Column.

Peak A corresponds to D-galactitol hexaacetate.
Oligosaccharide II was composed of two $(1 \rightarrow 4)$-linked D-glucose residues, one $(1 \rightarrow 3)$ linked D-galactose residue and one nonreducing terminal glucose residue (Table I). As the galactose residue should be located at the reducing end, the structure of oligosaccharide II must have the sequence $\beta$-D-GIc $P$ - $(1 \rightarrow 4)-\beta$ D-Glc $P$ - $(1 \rightarrow 4)-\beta$-D-Glc $P$ - $(1 \rightarrow 3)$-D-Gal $P$.

SG-D was composed of two $(1 \rightarrow 3)-$, two $(1 \rightarrow 4)$ - and two $(1 \rightarrow 6)$-linked D-glucose residues, one $(1 \rightarrow 3)$-linked D-galactose residue and one non-reducing terminal D-glucose residue with pyruvic acid (Table I), although no information about the binding site of succinic acid was obtained by this methylation method. 
Table 1. Methylation Analysis of Succinoglycan, Repeating Unit of Succinoglycan, Oligosaccharides I AND II and Their Derivatives

\begin{tabular}{|c|c|c|c|c|c|c|}
\hline & \multicolumn{6}{|c|}{ Methylated sugar (relative proportions) } \\
\hline & $\begin{array}{l}2,3,4,6-\mathrm{Glc} \\
\left(T=1.00^{a}\right)\end{array}$ & $\begin{array}{c}2,4,6-\mathrm{Glc} \\
\left(T=1.39^{a}\right)\end{array}$ & $\begin{array}{l}2,4,6-\mathrm{Gal} \\
\left(T=1.48^{a}\right)\end{array}$ & $\begin{array}{c}2,3,4-\mathrm{Glc} \\
\left(T=1.56^{a}\right)\end{array}$ & $\begin{array}{c}2,3,6-\mathrm{Glc} \\
\left(T=1.64^{a}\right)\end{array}$ & $\begin{array}{c}2,3-\mathrm{Glc} \\
\left(T=2.11^{a}\right)\end{array}$ \\
\hline Succinoglycan & 0 & 1.9 & 0.9 & 0.9 & 2.1 & 2.0 \\
\hline $\begin{array}{l}\text { Depyruvylated } \\
\text { succinoglycan }\end{array}$ & 1.0 & 2.1 & 1.1 & 1.0 & 2.3 & 1.1 \\
\hline $\begin{array}{l}\beta \text {-Glucosidase digested, } \\
\text { depyruvylated, desuccinylated } \\
\text { succinoglycan }\end{array}$ & 1.0 & 0.2 & 1.0 & 0.3 & 2.3 & 1.2 \\
\hline $\begin{array}{l}\text { Octasaccharide of } \\
\text { succinoglycan (SG-D) }\end{array}$ & 0 & 2.3 & 0.8 & 2.2 & 2.1 & 1.0 \\
\hline Depyruvylated SG-D & 1.0 & 2.2 & 0.7 & 2.1 & 2.0 & 0 \\
\hline $\begin{array}{l}\text { Desuccinylated } \\
\text { oligosaccharide I }\end{array}$ & 0 & 1.9 & 0 & 0.8 & $<0.1$ & 1.0 \\
\hline $\begin{array}{l}\text { Depyruvylated, } \\
\text { desuccinylated } \\
\text { oligosaccharide I }\end{array}$ & 1.0 & 2.1 & 0 & 0.8 & $<0.1$ & 0 \\
\hline Oligosaccharide II & 1.0 & $<0.1$ & 0.9 & 0 & 2.4 & 0 \\
\hline
\end{tabular}

a Retention time of the corresponding alditol acetate relative to 1,5-di- $O$-acetyl-2,3,4,6-tetra- $O$-methyl-D-glucitol. Column; $0.3 \%$ OV $275-0.4 \%$ GEXF1150 on Shimalite W.

Consequently, desuccinylated oligosaccharide I and II are linked through a $(1 \rightarrow 6) \mathrm{D}$-glucosidic linkage to form desuccinylated SG-D as follows.
It is understandable that intracellular $\beta$-glycanase (endo- $\beta$-( $(1 \rightarrow 6)$-D-glucanase) splits the $\beta$-D-Glc $P$ - $(1 \rightarrow 6)-\beta-\mathrm{D}-\mathrm{Glc} P$ bond in SG-D.

SG-D should be polymerized between $D$ -

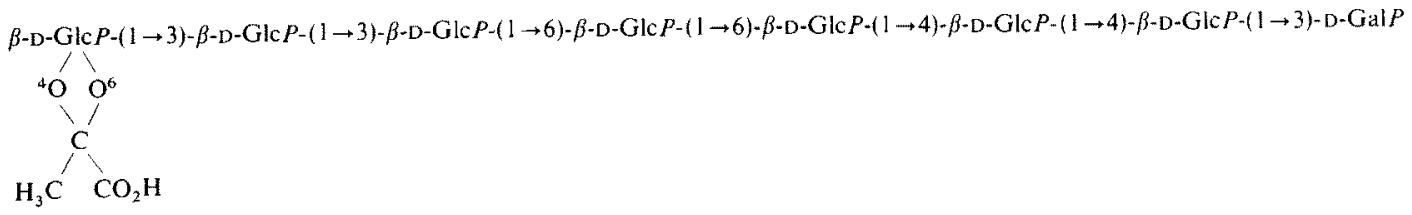

galactose and $(1 \rightarrow 6)$-linked $\mathrm{D}$-glucose residues through an intermolecular $(1 \rightarrow 4)$-linkage to form a branching structure. In order to determine which of the two $(1 \rightarrow 6)$-linked D-glucose residues in SG-D is concerned with the branching, depyruvylated, desuccinylated succinoglycan was digested with almond $\beta$-Dglucosidase, and the resulting digested polysaccharide was methylated. As shown in Table I, this polysaccharide lacks $90 \%$ of the $(1 \rightarrow 3)$ linked D-glucose and $70 \%$ of the $(1 \rightarrow 6)$-linked
D-glucose residues of the undigested polysaccharide. This indicates that two $(1 \rightarrow 3)$ - and one $(1 \rightarrow 6)$-linked D-glucose residues in SG-D locate at the side chain of succinoglycan and another $(1 \rightarrow 6)$-linked D-glucose residue locates at the branching point.

Since succinic acid is contained in oligosaccharide $\mathrm{I}^{10)}$ it should be linked to one of $\mathrm{D}$ glucose residues in the side chain of the polysaccharide although the location has not been clarified. From these results, the structure of 


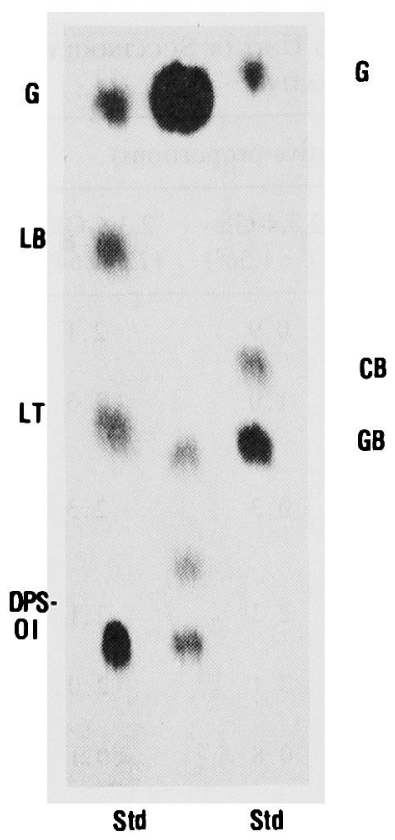

FIG. 4. Paper Chromatogram of the Products Formed by Partial Hydrolysis of Depyruvylated, Desuccinylated Oligosaccharide I with $\beta$-D-Glucosidase of Almond.

Std, standard mixtures; G, glucose; LB, laminarabiose; LT, laminaratriose; DPS-OI, depyruvylated, desuccinylated oligosaccharide I; CB, cellobiose; GB, gentiobiose.

succinoglycan was concluded to be as shown in Fig. 5.

The structure of succinoglycan elucidated here is identical, except for the occurrence of succinyl residue, with that of the polysac- charide from Rhizobium meliloti U27 investigated by Jansson et al. who used a method involving sequential degradation, methylation and modified Smith degradation. ${ }^{1)}$ The method including sequential fragmentation with the specific enzymes described here seems very good for elucidating the structures of complicated $\beta$-D-glycans like succinoglycan. The polysaccharides from $R h$. meliloti $\mathrm{U} 27$ and several strains of $\mathrm{Ag}$. radiobacter, Ag. rhizogenes and $A g$. tumefaciens were also digested successively with these two $\beta$-D-glycanases. The similarity of the chromatographic patterns of the products obtained has been reported in the separate paper. ${ }^{7)}$ The methylated sugars of each polysaccharide and the octasaccharide repeating unit were analyzed by GLC. As shown in Table II the relative proportions of all five methylated sugars of the polysaccharides and the octasaccharide repeating unit were quite similar to those of succinoglycan and the octasaccharide repeating unit from succinoglycan. Thus, these polysaccharides were principally identical. The polymers were partially different in the ratio of contents of succinyl or acetyl radical as shown already. ${ }^{31}$

Acknowledgments. This work was supported by grant 356076 to T.H. from the Ministry of Education and Culture of Japan. We thank Prof. B. Lindberg and Dr. R.E. Jansson in Stockholm University for a gift of polysac-

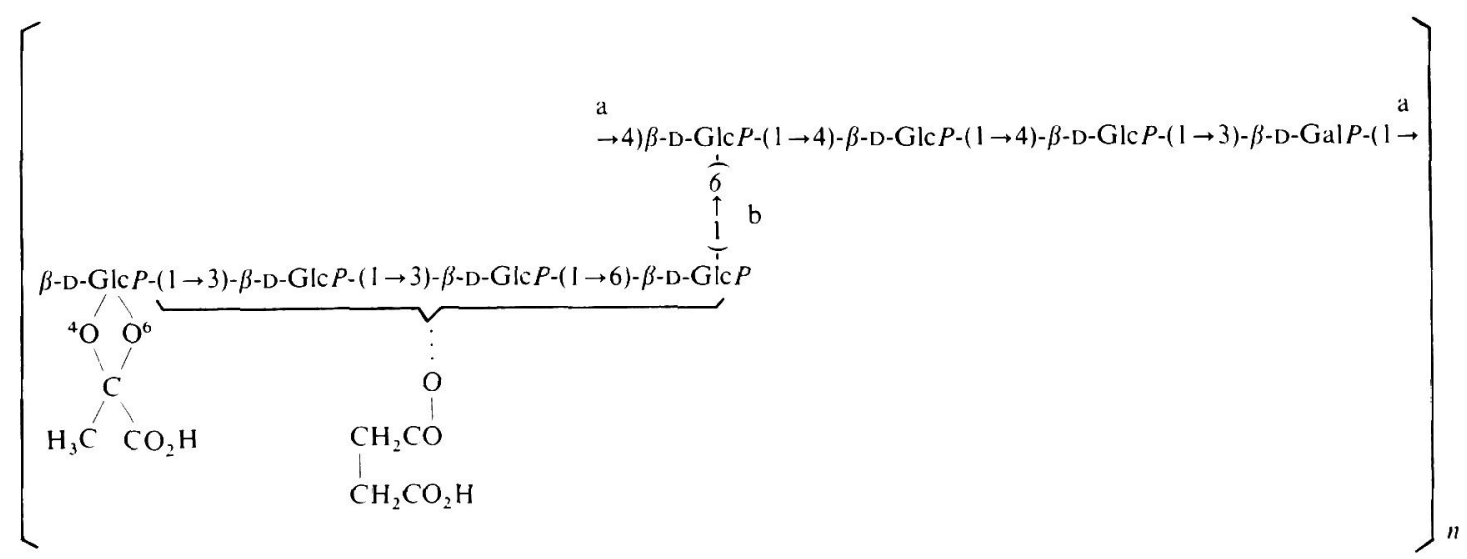

FIG. 5. Structure of Succinoglycan.

Linkages $\mathrm{a}$ and $\mathrm{b}$ are hydrolyzed by succinoglycan depolymerase and the intracellular $\beta$-D-glycanase, respectively. 
Table 11. Methylation Analysis of the Extracellular Acidic Polysaccharides (PS) From STRans of Alcaligenes, Agrobacterium AND Rhizobium, AND OF THEIR

Products (OS) Obtained With Succinoglycan Depolymerase

\begin{tabular}{|c|c|c|c|c|c|c|}
\hline & \multicolumn{6}{|c|}{ Methylated sugar (relative proportions) } \\
\hline & $2,3,4,6-\mathrm{Glc}$ & $2,4,6-\mathrm{Glc}$ & $2,4,6-\mathrm{Gal}$ & $2,3,4-G l c$ & $2,3,6-\mathrm{Glc}$ & 2,3-Glc \\
\hline \multicolumn{7}{|c|}{ Al. faecalis var. myxogenes } \\
\hline $10 \mathrm{C} 3 \mathrm{PS}$ & 0 & 1.9 & 0.9 & 0.9 & 2.1 & 2.0 \\
\hline $10 \mathrm{C} 3 \mathrm{OS}(\mathrm{SG}-\mathrm{D})$ & 0 & 2.3 & 0.8 & 2.2 & 2.1 & 1.0 \\
\hline \multicolumn{7}{|l|}{ Ag. radiobacter } \\
\hline IFO 12665 PS & 0 & 2.3 & 1.2 & 0.8 & 2.4 & 2.0 \\
\hline IFO 12665 OS & 0 & 2.0 & 0.8 & 1.9 & 2.1 & 1.0 \\
\hline \multicolumn{7}{|l|}{ Ag. rhizogenes } \\
\hline IFO 13259 PS & 0 & 1.8 & 0.9 & 0.7 & 2.0 & 2.0 \\
\hline IFO 13259 OS & 0 & 2.3 & 0.8 & 2.3 & 2.3 & 1.0 \\
\hline \multicolumn{7}{|l|}{ Ag. tumefaciens } \\
\hline IFO 3058 PS & 0 & 1.8 & 1.0 & 0.8 & 2.2 & 2.0 \\
\hline IFO 3058 OS & 0 & 2,4 & 0.9 & 2.2 & 2.4 & 1.0 \\
\hline \multicolumn{7}{|l|}{ Rh. meliloti } \\
\hline U $27 \mathrm{PS}$ & 0 & 1.8 & 1.0 & 0.8 & 2.0 & 2.0 \\
\hline U 27 OS & 0 & 2.3 & 0.9 & 2.4 & 2.2 & 1.0 \\
\hline
\end{tabular}

Column; $0.3 \%$ OV $275-0.4 \%$ GEXF 1150 on Shimalite W.

charide from Rh. meliloti $\mathrm{U} 27$. We are also indebted to Prof. B. Lindberg for his valuable advices.

\section{REFERENCES}

1) P.-E. Jansson, L. Kenne, B. Lindberg. H. Ljunggren, J. Lönngren, U. Rudén and S. Svensson, J. Am. Chem. Soc., 99, 3812 (1977).

2) T. Harada, Arch. Biochem. Biophys., 112, 65 (1965).

3) M. Hisamatsu, K. Sano, A. Amemura and T. Harada, Carbohydr. Res., 61, 89 (1978).

4) A. Misaki, H. Saito, T, Ito and T. Harada, Biochemistry, 8, 4645 (1969).

5) H. Saito, A. Misaki and T. Harada, Agric. Biol. Chem., 34, 1683 (1970).

6) L. P. T. M. Zevenhuizen, Carbohydr. Res., 26, 409 (1973).

7) T. Harada, A. Amemura, P.-E. Jansson and B. Lindberg, Carbohydr. Res., 77, 285 (1979).

8) T. Harada, K. Moori and A. Amemura, Agric. Biol.
Chem., 36, 2611 (1972).

9) A. Amemura, K. Moori and T. Harada, Biochim. Biophys. Acta, 334, 398 (1974).

10) J. Abe, A. Amemura and T. Harada, Agric. Biol. Chem., (Submitted).

11) T. Harada, T. Yoshimura, H. Hidaka and A. Koreeda, Agric. Biol. Chem, 29, 757 (1965).

12) A. Amemura and T. Harada, J. Ferment. Technol., 49 , 559 (1971).

13) S. Hakomori, J. Biochem. (Tokyo), 55, 205 (1964).

14) B. Lindberg, Methods in Enzymology, Vol. 28, ed by V. Ginsburg, Academic Press Inc., New York, 1972, p. 183.

15) T. Yamada, M. Hisamatsu and M. Taki, Nippon Nôgeikagaku Kaishi, 49, 163 (1975).

16) J. H. Sloneker and A. Jeanes, Can. J. Chem., 40, 2066 (1962).

17) A. S. Chaudhari, C. T. Bishop and W. F. Dudman, Carbohydr. Res., 28, 221 (1973). 